\title{
Geomagnetically trapped anomalous cosmic rays
}

\author{
R. S. Selesnick, A. C. Cummings, J. R. Cummings, R. A. Mewaldt, and \\ E. C. Stone \\ California Institute of Technology, Pasadena
}

T. T. von Rosenvinge

NASA Goddard Space Flight Center, Greenbelt, Maryland

\begin{abstract}
Since its launch in July 1992, the polar-orbiting satellite SAMPEX has been collecting data on geomagnetically trapped heavy ions, predominantly $\mathrm{O}, \mathrm{N}$, and $\mathrm{Ne}$, at energies $\gtrsim 15 \mathrm{MeV} /$ nucleon and in a narrow $L$ shell range near $L=2$. Their location, elemental composition, energy spectra, pitch angle distribution, and time variations all support the theory that these particles originated as singly ionized interplanetary anomalous cosmic rays that were stripped of electrons in the Earth's upper atmosphere and subsequently trapped. The $\mathrm{O}$ are observed primarily at pitch angles outside the atmospheric loss cones, consistent with a trapped population, and their distribution there is nearly isotropic. The abundances relative to 0 of the $\mathrm{N}$, possibly $\mathrm{Ne}$, and especially $\mathrm{C}$ are lower than the corresponding interplanetary values, which may be indicative of the trapping efficiencies. The distributions of trapped N, O, and Ne in energy and $L$ shell suggest that most of the ions observed at the SAMPEX altitude of $\sim 600 \mathrm{~km}$ are not fully stripped when initially trapped. A comparison of the trapped intensity with the much lower interplanetary intensity of anomalous cosmic rays provides model-dependent estimates of the product of the trapping probability and the average trapped particle lifetime against ionization losses in the residual atmosphere for particles that mirror near the SAMPEX altitude.
\end{abstract}

\section{Introduction}

Interplanetary anomalous cosmic rays (ACRs) are observed as low-energy ( 10 to $50 \mathrm{MeV} /$ nucleon) increases in the flux of the elements $\mathrm{He}, \mathrm{C}, \mathrm{N}, \mathrm{O}, \mathrm{Ne}$, $\mathrm{Ar}$, and possibly $\mathrm{H}$ at times of low solar modulation [McDonald et al., 1974; Garcia-Munoz et al., 1973; Hovestadt et al., 1973; Christian et al., 1989; Cummings and Stone, 1990]. They are thought to result from interstellar neutral particles that drift into the heliosphere, become ionized by solar ultraviolet radiation or charge exchange with the solar wind, are picked up and convected outward by the solar wind, and are accelerated in the outer heliosphere [Fisk et al., 1974], probably at the solar wind termination shock [Pesses et al., 1981]. Evidence from several sources [e.g., Adams et al., 1991; Singh et al., 1991; and references therein], particularly the recent results from SAMPEX [Klecker et al., 1994], have now verified the prediction that the ACRs are singly ionized, unlike the galactic cosmic ray and solar energetic particle components that are totally stripped or nearly so.

Copyright 1995 by the American Geophysical Union.

Paper number 94JA03140.

0148-0227/95/94JA-03140\$05.00
The possibility that interplanetary ACRs can be trapped in the geomagnetic field was proposed by Blake and Friesen [1977]. Theoretical predictions of the trapped ACR spatial distributions and energy spectra have since been updated and refined [Blake, 1990; Tylka, 1994; M. Schulz et al., unpublished manuscript, 1993]. Observations of trapped heavy ions with passive track detectors on Skylab were made by Chan and Price [1975] and by Biswas et al. [1975]. Biswas and Durgaprasad [1980] suggested that these were the trapped ACRs predicted by Blake and Friesen. However, because the composition observed on Skylab included substantial abundances of $\mathrm{C}$ and heavier elements such as $\mathrm{Fe}$, other possible origins such as the solar wind were also indicated [Chan and Price, 1975; Biswas and Durgaprasad, 1980]. The first strong observational evidence in support of the theory was inferred from data taken by the Kosmos satellites with passive track detectors [Grigorov et al., 1991]. The composition, angular distribution, and temporal variations of the Kosmos data were consistent with a trapped ACR population, although the track detectors did not provide direct measurements of the ions' spatial distribution. Confirmation that they were in a region of stable geomagnetic trapping came from measurements of $\mathrm{N}, \mathrm{O}$, and $\mathrm{Ne}$ ions at kinetic energies of $\gtrsim 15 \mathrm{MeV} /$ nucleon, with spatial and temporal resolution, by the mass spectrometer telescope (MAST) 
on the polar-orbiting SAMPEX satellite [Cummings et al., 1993, 1994].

In this paper we continue the analysis of the MAST data taken during the time period starting from the SAMPEX launch in July 1992 and ending in February 1994. We provide quantitative estimates of the trapped particle intensity, spatial distribution, and energy spectra, and we relate these estimates to the interplanetary ACR intensity. In section 2 we introduce the parameters thought to control the trapping of ACRs and present the MAST data in this context. In section 3 we describe the observed composition of the trapped ACRs and its variation with energy. Section 4 contains a detailed calculation of the intensity of trapped ACR oxygen as a function of energy, pitch angle, time, and $L$ shell. A model relating the intensities of interplanetary and trapped ACRs is described in section 5, from which estimates of the trapping probability and the average trapped particle lifetime are derived. The major conclusions of this study are summarized in section 6 .

\section{ACR Trapping}

\subsection{Trapping Conditions}

The Blake and Friesen [1977] model was based on the prediction that the interplanetary ACRs are singly ionized. Upon reaching the upper atmosphere at midlatitudes, they may be stripped of some or all of their remaining electrons, thus undergoing an abrupt change in magnetic rigidity. The change is such that the subsequent particle motions are more tightly constrained by the geomagnetic field, and under the right circumstances, they will remain trapped.

The access of singly ionized interplanetary ACRs to low magnetic $L$ shells is governed by the geomagnetic cutoff. In a dipole field the cutoff rigidity for a particle arriving from the west, which is lower than for other arrival directions, is

$$
\mathcal{R}_{c w}=\frac{C_{s}}{L^{2}\left[1+\left(1+(r / L)^{3 / 2}\right)^{1 / 2}\right]^{2}}
$$

where $r$ is the distance from the dipole center in Earth radii and $C_{s}=58 \mathrm{GV}$ for the Earth's magnetic dipole moment in 1990 [Smart and Shea, 1993]. Particles that arrive at rigidities just above cutoff and are stripped of some or all of their remaining electrons by the Earth's upper atmosphere have the potential to become trapped. The western cutoff is appropriate because, after stripping, these particles are at a rigidity below cutoff and are also moving approximately perpendicular to the local magnetic field. Then they are near the mirror point of any subsequent trapped particle trajectory, so that they may not be lost in the atmosphere as would probably be the case for a particle arriving from a nearvertical direction.

Whether or not the particle is trapped is determined by the value of the adiabaticity parameter $\epsilon$, which is the ratio of the particle gyration and bounce periods. Theoretical estimates of the maximum value of $\epsilon$ al- lowed for a trapped orbit vary from $1 / 3$ [Schulz, 1991, p. 202 ] to $\sim 0.1$ [Ilyan et al., 1986]. For a particle of rigidity $\mathcal{R}$ in a dipole magnetic field, $\epsilon=3 \mathcal{R} /\left(L a B_{0}\right)$, where $a$ is the radius of the Earth and $B_{0}$ is the equatorial magnetic field for a given $L$ shell. With the Earth's dipole moment of $7.84 \times 10^{15} \mathrm{Tm}^{3}$

$$
\epsilon=5.18 \times 10^{-5} \frac{A}{Q}[E(E+1863)]^{1 / 2} L^{2}
$$

where $E$ is the kinetic energy in $\mathrm{MeV}$ per nucleon, $A$ is the atomic mass number, and $Q$ is the charge state.

As a first illustration of the data obtained by MAST, particle distributions in energy versus $L$ shell with solar active periods eliminated are shown in Figure 1. Also shown are curves defined by the western cutoff rigidity, according to (1), for both singly ionized and fully stripped atoms, and a value of $\epsilon Q=0.8$ based on (2). The $\epsilon Q$ value was chosen empirically (see further discussion below) to represent an upper bound on the trapped particle locations. The elements shown in Figure 1 were selected to include four normally found in the ACR component ( $\mathrm{C}, \mathrm{N}, \mathrm{O}$, and $\mathrm{Ne})$, and two that are not $(\mathrm{Mg}$ and $\mathrm{Fe})$. The elements are easily distinguished in the data because MAST was designed to resolve isotopes of cosmic ray elements from $\mathrm{He}$ to $\mathrm{Ni}$ and to provide accurate measurements of each particle's energy and arrival direction [Cook et al., 1993].

The non-ACR elements in Figure 1 were generally detected at locations above the cutoff for fully stripped ions and are all well above the cutoff for singly ionized atoms. Some of the data are slightly below the fully stripped particle cutoff, either suggesting that these particles were only partially ionized and perhaps of solar origin, or reflecting inaccuracies in the nominal cutoff locations [Fanselow and Stone, 1972]. The second possibility appears to be more likely because data taken from the large solar flare events of October and November 1992 [Selesnick et al., 1993] show that solar C ions, most of which are presumably fully stripped, also follow a cutoff that is below the nominal dipole value and consistent with the data in Figure 1. However, the $\mathrm{Mg}$ and $\mathrm{Fe}$ data strongly support a lack of singly ionized atoms for these elements, and there is no evidence for any trapped populations.

The presence of singly ionized atoms is clearly indicated in Figure 1 for the ACR elements $\mathrm{N}, \mathrm{O}$, and $\mathrm{Ne}$ by the distribution of relatively low energy particles between the cutoffs for singly ionized and fully stripped particles. The $\mathrm{C}$ data show only a small number of particles that may be singly ionized, which is consistent with the small $\mathrm{C}$ contribution to the interplanetary ACRs (a detailed comparison of the relative elemental abundances is given in section 3 ). In addition to the diffuse population of ACR ions that are observed between the two cutoffs in Figure 1, there is a more intense and localized band of particles near the lower cutoff. This is particularly evident for $\mathrm{O}$, but also for $\mathrm{N}$ and $\mathrm{Ne}$. These are the particles that have previously been identified as geomagnetically trapped ACRs [Cummings et al., 1993, 1994]. 


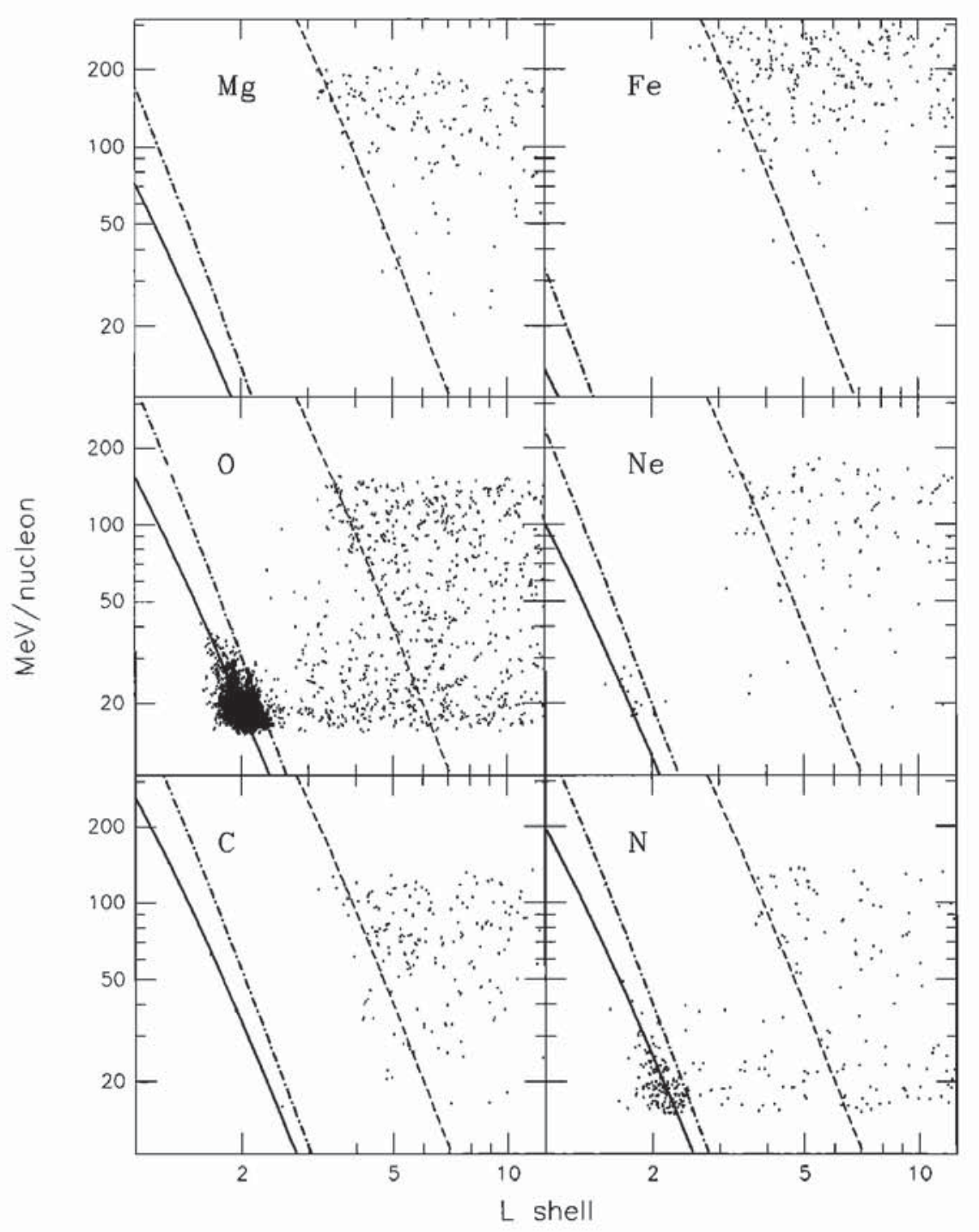

Figure 1. The observed solar quiet-time particle distribution in energy per nucleon versus $L$ shell for selected elements from July 1992 to February 1994 . The curves represent model geomagnetic west cutoffs for singly ionized (solid curves) and fully stripped (dashed curves) atoms and a value for the trapping parameter times charge state of $\epsilon Q=0.8$ (dash-dot curves). The energy range of MAST varies from $\sim 16-160 \mathrm{MeV} /$ nucleon for $\mathrm{O}$ to $\sim 28-310 \mathrm{MeV} /$ nucleon for Fe.

Immediately after an ACR is stripped of electrons by the atmosphere and becomes trapped, it is at a location where the rigidity of a singly ionized particle of the same energy would be above the western cutoff rigidity. The lifetime of the trapped particle is then determined by energy loss in the residual atmosphere, with pitch angle and radial diffusion being negligible on this timescale [Schulz and Lanzerotti, 1974]. The existence of trapped $\mathrm{O}$ and $\mathrm{N}$ below the nominal lower cutoff is therefore probably a result of such energy loss. In this model, no particles should be observed at $L$ values lower than the location of the cutoff for the highest-energy trapped particles. The particles should then fill in all energies below cutoff and at each allowed $L$ value as they lose energy. The data appear to be at least qualitatively consistent with such an interpretation.

The upper limit in $L$, at which the particles may remain trapped immediately after they are initially stripped of electrons by the atmosphere is determined by the $\epsilon$ parameter, which depends on the initial charge state $Q$ as described above. If the trapped particles are not fully stripped initially, then further reductions in $Q$ are likely on subsequent encounters with the upper atmosphere. Since $Q$ at the time of trapping is not known we can only calculate the combination $\epsilon Q$ from (2). Histograms of $\epsilon Q$ for all of the quiet time $\mathrm{N}, \mathrm{O}$, and $\mathrm{Ne}$ are shown in Figure 2. They illustrate the confinement of the trapped particles in a more quantitative fashion and the low backgrounds of interplanetary ACRs at higher $\epsilon Q$ values. Figure 2 shows that the maximum value of $\epsilon$ for each trapped component is related to the initial charge state by $\epsilon Q \approx 0.8$, which corresponds to the curves shown in Figure 1 . In the following analyses we define the trapped ACR populations as those particles with $\epsilon Q<1.0$ and include all data from the time period July 1992 to February 1994 , because the trapped ACRs are not contaminated by solar particles during solar active times. 


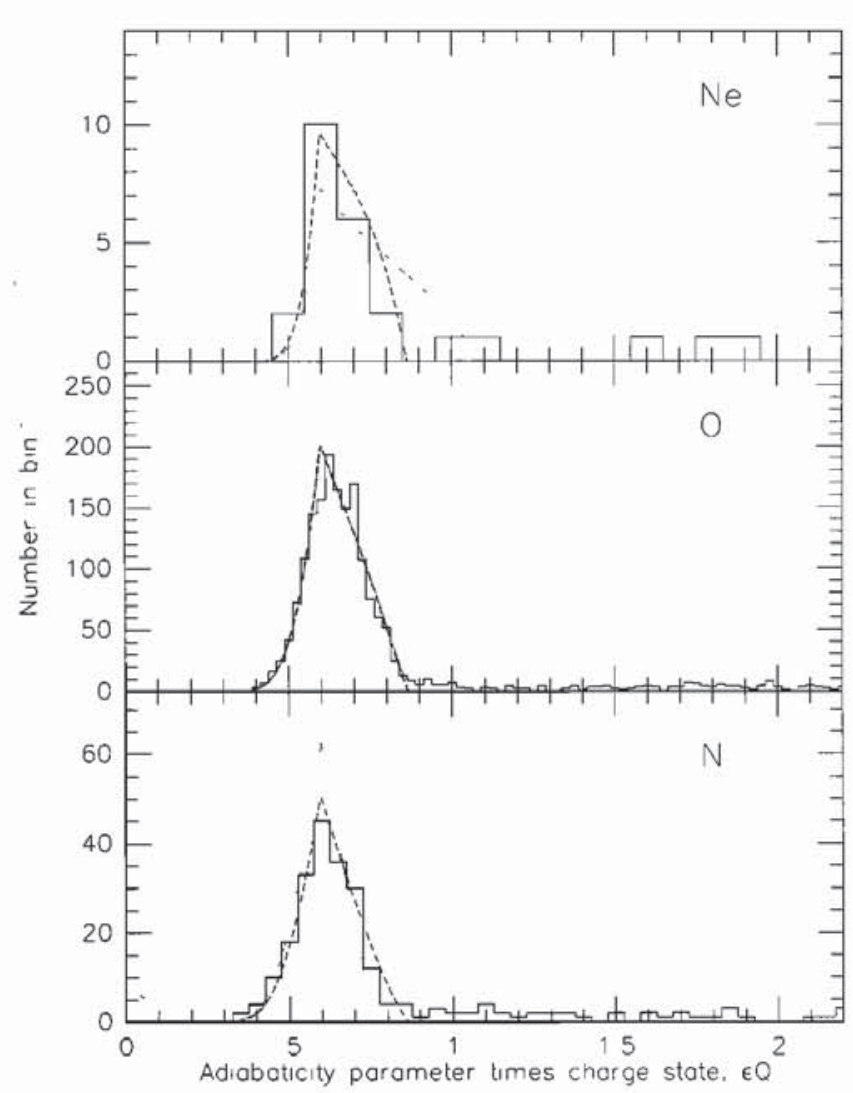

Figure 2. Histograms of the trapping parameter $\epsilon$ times initial charge state $Q$ for the solar quiet time $\mathrm{N}, \mathrm{O}$, and $\mathrm{Ne}$ data. Note the different bin widths for each element. The dashed and dotted curves show model simulations based on the respective assumptions of identical initial charge states and fully stripped initial charge states.

\subsection{Inferred Charge States}

Both Cummings et al. [1993] and Tylka [1994] derived a maximum $\epsilon$ value of $\sim 0.1$ from the trapped $\mathrm{O}$ distribution and the assumption that the ions are fully stripped. The $O$ histogram in Figure 2, with the assumption that $Q=8$, is consistent with this result. However, as will be shown below, the $\mathrm{N}, \mathrm{O}$, and $\mathrm{Ne}$ histograms taken together do not appear to be consistent with the fully stripped hypothesis and with a single maximum $\epsilon$ value. Since we expect that a single maximum $\epsilon$ value should apply to all elements, the assumption that all elements are fully stripped at the time of trapping may be incorrect. Blake and Friesen [1977] show that particles with mirror points at $300 \mathrm{~km}$ altitude are likely to be stripped of all electrons, but this is probably not true if the mirror points are at the SAMPEX altitude of $\sim 600 \mathrm{~km}$. In fact, the trapped particles observed by SAMPEX are likely to have a combination of initial charge states due to their varying mirror point altitudes. Detailed theoretical modeling will be required to determine the expected charge state values. However, some constraints can be obtained from the data.
To illustrate these considerations in a quantitative way, we have calculated expected $\epsilon Q$ distributions based on the model intensity as a function of $E$ and $L$ that is described in section 5 below. The calculations take into account the observed energy spectra of the trapped ACRs for each element (see section 3 below), the variation in geomagnetic cutoff with $L$, a single maximum $\epsilon$ value for all elements, energy loss in the residual atmosphere after trapping, the energy thresholds of MAST for each element, and the variation in observing time as a function of $L$. At each $\epsilon Q$ value the model intensity is numerically integrated over $L$ to simulate the histograms in Figure 2. Five parameters are adjusted to give a best fit to the data: multiplicative normalization factors for each of the three elements, the maximum epsilon value, and the constant (1) for the geomagnetic cutoff. The results are shown by the curves in Figure 2 for two cases. The dotted curve corresponds to a maximum $\epsilon$ value of 0.1082 and fully stripped initial charge states for each element. The dashed curve corresponds to a common initial $Q$ for each element and a maximum $\epsilon$ value of $0.8625 / Q$. In each case the best fit was obtained by reducing the western cutoff rigidity by $5 \%$ from its nominal dipole value.

The two models in Figure 2 are nearly equivalent for $O$ because it provides the dominant constraint on the fits due to its higher number of counts. The models are different for $\mathrm{N}$ and $\mathrm{Ne}$, with the assumption of common charge states producing a slightly better fit in each case. The $\chi^{2}$ function (defined in terms of the Poisson likelihood function in an equivalent way to the standard Gaussian $\chi^{2}$ ) for 34 data points from all three elements is 94.8 , compared to a value of 115.1 for the fully stripped case. The contributions to the $\chi^{2}$ values from only the $20 \mathrm{O}$ data points are 47.1 and 48.4 , respectively, while those from a combination of the 14 $\mathrm{N}$ and $\mathrm{Ne}$ data points are 47.7 and 66.7 , respectively, so the difference between the two cases is primarily due to the $\mathrm{N}$ and $\mathrm{Ne}$ data points included in the fits. Although the model has some simplifying assumptions which cause the $\chi^{2}$ values to exceed those expected from Poisson statistics, it does show that the data may be more consistent with similar average charge states for each element. Finally, if we assume that the elements are initially stripped down to their 1s electrons, with charge states of 5 for $\mathrm{N}, 6$ for $\mathrm{O}$, and 8 for $\mathrm{Ne}$, then a worse fit to the data is obtained than in the fully stripped case because of the larger relative differences in the initial charge states.

Some additional information on the trapping process can be obtained by considering $\mathrm{He}$ and $\mathrm{C}$. Although trapped He has been observed by SAMPEX, it is not thought to be of ACR origin [Cummings et al., 1993, 1994]. Therefore stripping a singly ionized ACR He ion to its maximum charge state of $Q=2$, or decreasing the rigidity of any ion by a factor of 2 , is apparently insufficient to cause trapping. The lack of trapped He therefore implies $Q>2$ for all trapped ACRs. The limit $\epsilon Q \lesssim 0.86$ from Figure 2 then gives $\epsilon \lesssim 0.29$. 


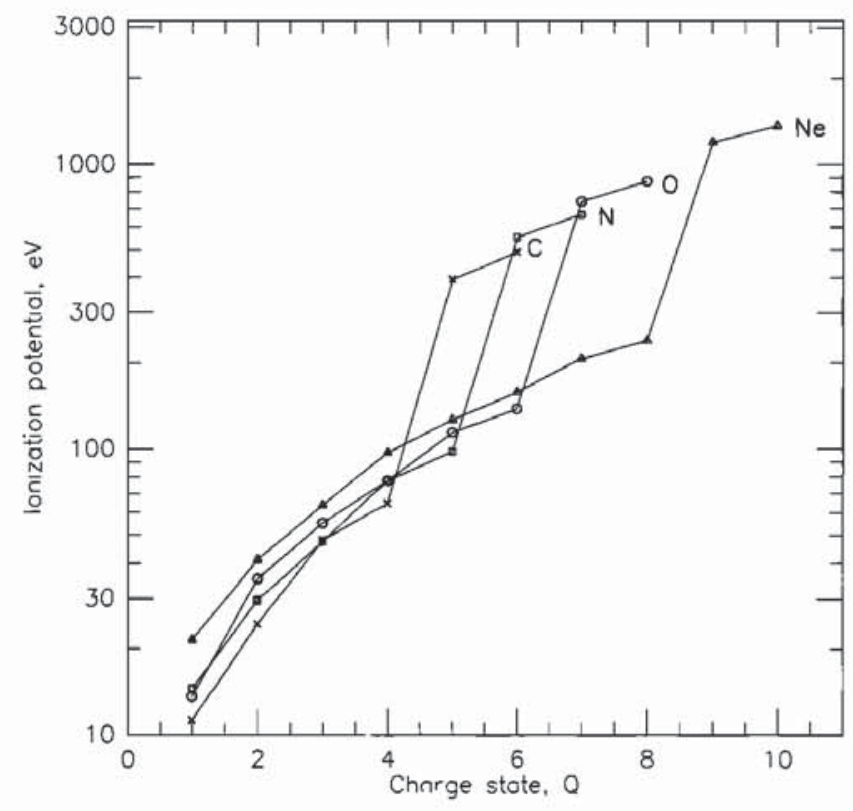

Figure 3. Ionization potentials for each of the charge states of $\mathrm{C}, \mathrm{N}, \mathrm{O}$, and $\mathrm{Ne}$.

A similar argument can be made based on the low abundance of trapped $\mathrm{C}$ (see below). The rigidity of ACR C can decrease by a maximum factor of 6 if its charge state changes from 1 to 6 . This may suggest $Q>6$ for all trapped ACRs, and $\epsilon \lesssim 0.12$, with perhaps a small efficiency for trapping $Q \stackrel{\sim}{=} 6$ to account for the small fraction of trapped C. However, it is also important to consider possible variations in the stripping cross sections for different elements and charge states. Although these are not well known [Spjeldvik, 1979], they are likely to be controlled to a large extent by the ionization potentials, which are shown for reference in Figure 3. Because the ionization potentials for the two inner (1s) electrons are relatively high, it may be that the maximum initial charge state for $\mathrm{C}$ is effectively $Q=4$. Then the limits for $\mathrm{N}, \mathrm{O}$, and Ne would be $Q>4$ and $\epsilon \lesssim 0.17$. One might expect that $\mathrm{N}, \mathrm{O}$, and Ne would also be stripped down to their 1s electrons due to their similarly high ionization potentials, although as was noted above, this does not provide the best fit to the data. Although we cannot uniquely determine from the data the maximum $\epsilon$ value or the initial $Q$ of the trapped ACRs, the results described above suggest that the simple picture of fully stripped initial charge states and a single maximum $\epsilon$ value may not be valid at SAMPEX altitudes.

As an aside, if $\epsilon$ has a maximum value of $\sim 0.15$ to $\mathbf{0 . 2}$, as may be consistent with the observations reported here, then there is an improved possibility of trapping heavy solar energetic particles (SEPs) by the Blake and Friesen method. SEP $\mathrm{Mg}, \mathrm{Si}$, and $\mathrm{Fe}$ have been observed to have mean charge states of 10.8 , 11.0, and 14.1, respectively [Luhn et al., 1984], similar to typical solar wind values. Assuming a maximum $\epsilon$ of $1 / 9$, Kleis et al. [1993] concluded that ions must have $Q_{1} / Q_{2}<0.12$ to 0.15 to be trapped by the Blake and Friesen method, where $Q_{1}$ and $Q_{2}$ are the particle's charge states before and after stripping in the atmosphere. They further concluded that SEP $\mathrm{Mg}, \mathrm{Si}$, and $\mathrm{Fe}$ do not satisfy this requirement, even when the expected tails of the charge state distributions are considered, but suggested that such ions may be trapped during large geomagnetic storms when the geomagnetic cutoff is suppressed. Although there are presently no measurements of the distribution of SEP charge states, recent solar wind observations by Ulysses include charge states as low as +5 to +7 for $\mathrm{Fe}$ [Geiss et al., 1994]. If SEPs also contain these charge states, and if the maximum $\epsilon \sim 0.15$ to 0.2 , thereby relaxing the condition for trapping to $Q_{1} / Q_{2}<0.2$ to 0.25 , SEP Fe might be trapped by the Blake and Friesen method during geomagnetically quiet conditions.

\section{Composition}

A histogram of the atomic number $Z$ for the trapped particles with $Z>2$ is shown in Figure 4. Peaks for $\mathrm{N}, \mathrm{O}$, and $\mathrm{Ne}$ are clearly evident, although the elemental resolution is somewhat poorer in this region than at high geographic latitudes due to the high intensity of trapped protons. Three $\mathrm{C}$ events and one possible $\mathrm{B}$ were also observed. To compare the relative abundances of the elements in different energy ranges, integral energy spectra for trapped $\mathrm{C}, \mathrm{N}, \mathrm{O}$, and $\mathrm{Ne}$ are shown in Figure 5. Each point at a given energy represents the relative intensity of each element for all particles observed above that energy. The data were normalized by the average geometry factors for each energy bin, but the time interval, which is equal for each element and energy bin, was not taken into account (see section 4). The results in Figure 5 show that when compared on the basis of energy per nucleon $E$, the relative abundances of trapped $\mathrm{N}, \mathrm{O}$, and $\mathrm{Ne}$ vary with

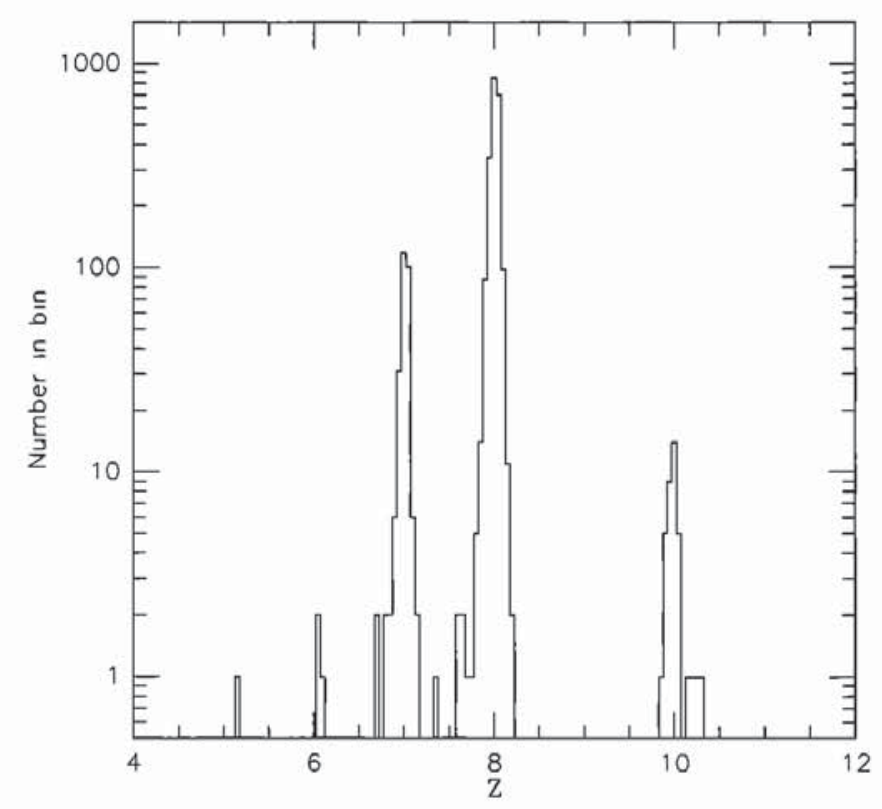

Figure 4. Histogram of the estimated nuclear charge (atomic number). The value of $\epsilon Q$ was required to be less than 1.0 to include only the trapped particle data. 


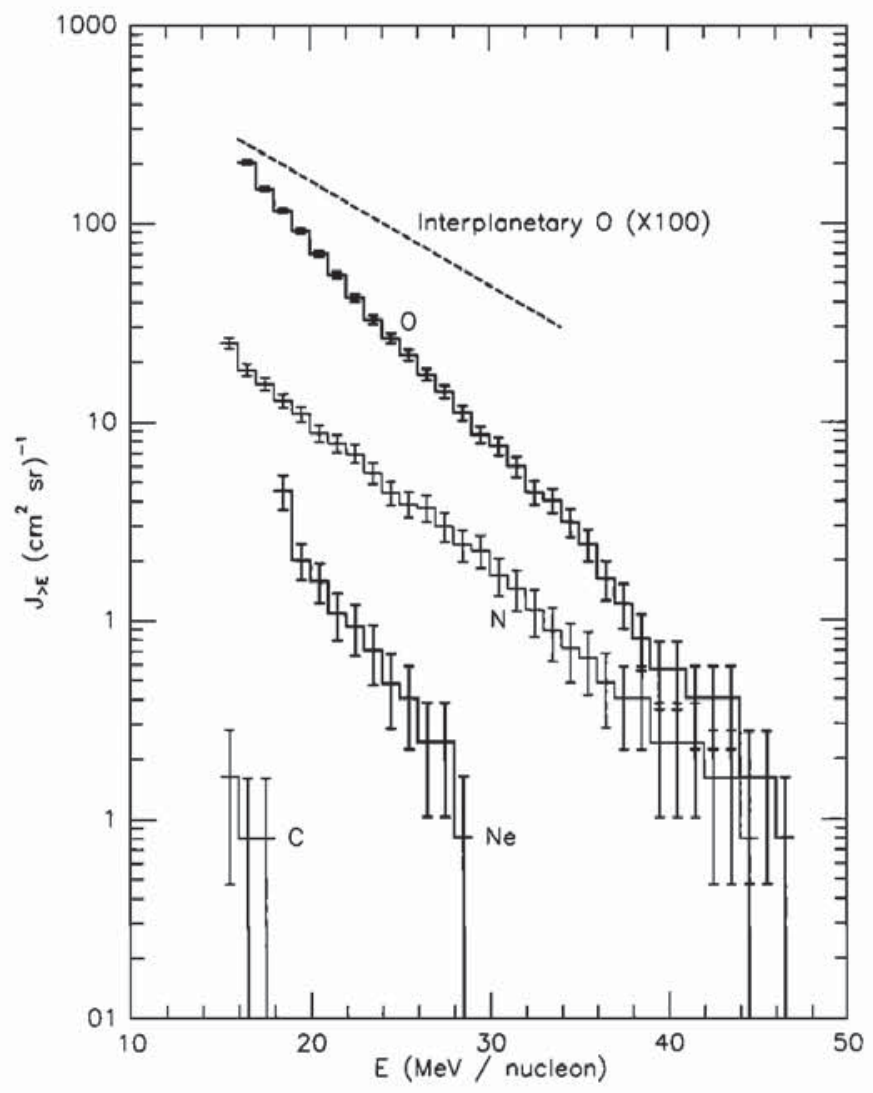

Figure 5. Integral energy spectra of trapped $\mathrm{C}, \mathrm{N}$, $\mathrm{O}$, and $\mathrm{Ne}$ fluences. The relative interplanetary $\mathrm{O}$ spectrum is also shown.

$E$ in such a way that the integral spectra soften with increasing $Z$. Interplanetary measurements of ACR $N$, $\mathrm{O}$, and $\mathrm{Ne}$ in the same energy range from Voyager 2 in 1987 do not show this effect [Cummings and Stone, 1990].

The trapped energy spectra, as shown in Figure 5, are significantly softer than the interplanetary spectra. For 0 , the trapped spectrum from $\sim 16$ to $45 \mathrm{MeV} /$ nucleon is consistent with an exponential and an $e$-folding energy of $\sim 4 \mathrm{MeV} /$ nucleon. The interplanetary $\mathrm{O}$ spectrum from $\sim 10$ to $30 \mathrm{MeV} /$ nucleon measured by Voyager 2 near $1 \mathrm{AU}$ in 1977-1978 [Cummings et al., 1984] had an $e$-folding energy of $\sim 8 \mathrm{MeV} /$ nucleon, and a similar result is obtained from the interplanetary SAMPEX data [Mewaldt et al., 1993], as indicated in Figure 5. Some softening of the trapped spectra relative to the interplanetary spectra is expected near the maximum trapped particle energy [Tylka, 1994], but a more likely explanation for the observed spectra may be a decrease with energy of the cross sections for stripping the interplanetary ACRs in the upper atmosphere [Spjeldvik, 1979].

The abundances relative to $\mathrm{O}$ of $\mathrm{C}, \mathrm{N}$, and $\mathrm{Ne}$, for the energy range from 16 to $30 \mathrm{MeV} /$ nucleon, are listed in Table 1 for both the trapped and interplanetary measurements from SAMPEX and the interplanetary measurements from Voyager 2 [Cummings and Stone, 1990]. As is shown in the table, trapped C, N, and possibly $\mathrm{Ne}$ are depleted relative to $\mathrm{O}$. For $\mathrm{N}$ the depletion is a factor of $\sim 2$. For $\mathrm{C}$ we should have seen $\sim 100$ trapped events if the $\mathrm{C} / \mathrm{O}$ abundance were equal to the interplanetary value at Voyager 2, instead of the three events that were seen. Similar results for $N$ and $\mathrm{Ne}$, although with somewhat lower abundances relative to O, were obtained by Bobrovskaya et al. [1993] from the Kosmos data at 10 to $20 \mathrm{MeV} /$ nucleon. Again, detailed modeling of the expected charge states and trapping processes will be required for a theoretical understanding of the relative abundances and energy spectra.

\section{Trapped Particle Intensities}

\subsection{Method of Calculation}

The intensity at the spacecraft $j(E, \alpha, L, t)$ can, in general, be described as a function of kinetic energy per nucleon, $E$; pitch angle $\alpha$ relative to the local magnetic field; magnetic $L$ shell; and time $t$. Each observed particle is characterized by its measured kinetic energy, nuclear charge, mass, trajectory through the telescope, and the time of observation. From these properties, and the information available about the spacecraft orbit and pointing directions, and the geomagnetic field model, it is straightforward to calculate $E, L$, and $\alpha$ (the International Geomagnetic Reference Field (IGRF) 1985 model with secular updates was used to calculate all magnetic field parameters). The local and equatorial values of $\alpha$ and the magnetic field magnitude $B$ are related by

$$
\frac{\sin ^{2} \alpha}{B}=\frac{\sin ^{2} \alpha_{0}}{B_{0}}
$$

where the subscript 0 denotes equatorial values. Ideally, one would calculate from the local measurements the

Table 1. Anomalous Cosmic Ray Abundance Ratios

\begin{tabular}{|c|c|c|c|}
\hline & \multicolumn{2}{|c|}{ SAMPEX (1992-1993) } & \multirow{2}{*}{$\begin{array}{c}\text { Voyager } 2 \text { (1987), } \\
\text { Interplanetary, } \\
\text { 16-30 MeV/nucleon }\end{array}$} \\
\hline & $\begin{array}{c}\text { Trapped, } \\
\text { 16-45 } \mathrm{MeV} / \text { nucleon }\end{array}$ & $\begin{array}{l}\text { Interplanetary, } \\
\text { 16-25 MeV/nucleon }\end{array}$ & \\
\hline $\begin{array}{l}\mathrm{C} / \mathrm{O} \\
\mathrm{N} / \mathrm{O} \\
\mathrm{Ne} / \mathrm{O}\end{array}$ & $\begin{array}{r}\sim 0.0004 \\
0.09 \pm 0.01 \\
0.04 \pm 0.01^{*}\end{array}$ & $\begin{array}{l}0.18 \pm 0.03 \\
0.08 \pm 0.02\end{array}$ & $\begin{array}{l}0.020 \pm 0.004 \\
0.194 \pm 0.013 \\
0.048 \pm 0.006\end{array}$ \\
\hline
\end{tabular}

"Lower energy limit for SAMPEX Ne/O is $18 \mathrm{MeV} /$ nucleon. 
equatorial intensity $j_{0}$. However, because the spacecraft orbits at low altitude (520 to $675 \mathrm{~km})$ there is only a small range of $\alpha_{0}$ near zero (and $180^{\circ}$ ) that is observable. Typical values of $B / B_{0}$ are $\sim 5$ to 6 . It is convenient to introduce a new set of variables $R$ and $\Lambda$, the dipole equivalent radius and magnetic latitude, that are related to $L$ and $B / B_{0}$ by

$$
\begin{gathered}
R=L \cos ^{2} \Lambda \\
\frac{B}{B_{0}}=\frac{L^{3}}{R^{3}}\left(4-\frac{3 R}{L}\right)^{1 / 2}
\end{gathered}
$$

(These are similar to the $R-\Lambda$ coordinates defined by Roederer [1970], who replaces $B_{0}$ by its dipole value $k_{0} /(L a)^{3}$, where $k_{0}$ is the Earth's dipole moment.) The data are taken at various $R$ and $\Lambda$ values along the SAMPEX orbit. By converting the measured pitch angles to the values that would be observed on the same $L$ shells at a single value of $R=R_{1}$ the pitch angle distribution is made similar to that actually observed and is easily converted to the equivalent distribution at any other point along the field line. The intensity $j_{1}$ at $R=R_{1}$ is related to the local intensity by Liouville's theorem $j_{1}\left(E, \alpha_{1}, L, t\right)=j(E, \alpha, L, t)$. The new pitch angle $\alpha_{1}$ is related to $\alpha$ by

$$
\sin ^{2} \alpha_{1}=\frac{B_{0}}{B} \frac{L^{3}}{R_{1}^{3}}\left(4-\frac{3 R_{1}}{L}\right)^{1 / 2} \sin ^{2} \alpha
$$

In addition, the loss-cone angles at different $R$ values are converted to nearly constant value of $\alpha_{1}$. We have adopted a value of $R_{1}=1.3$, which is near the upper limit of values reached by the spacecraft in the region where the data where observed, so that only a few $(\sim 5 \%)$ of the observed particles have their mirror points above this value of $R$ and are not included in the calculated intensities.

To calculate $j_{1}\left(E, \alpha_{1}, L, t\right)$ the data are divided into bins. The number of events, $N_{i k n m}$, observed in the $i$ th energy bin from $E_{i}$ to $E_{i+1}$, the $k$ th pitch angle bin from $\alpha_{1 k}$ to $\alpha_{1 k+1}$, the $n$th $L$ shell bin from $L_{n}$ to $L_{n+1}$, and the $m$ th time bin from $t_{m}$ to $t_{m+1}$ is related to $j_{1}$ by

$$
\begin{gathered}
N_{i k n m}=\int_{0}^{2 \pi} \int_{\alpha_{2 k}}^{\alpha_{1 k+1}} \int_{E_{v_{v}}}^{E_{i+1}} \int_{\substack{t_{m} \\
L_{n}<L<L_{n+1}}}^{t_{m+1}} A(E, \theta(\alpha, \beta), \phi(\alpha, \beta)) \\
\cdot \cos \theta(\alpha, \beta) j_{1}\left(E, \alpha_{1}, t, L\right) \sin \alpha \frac{d \alpha}{d \alpha_{1}} d t d E d \alpha_{1} d \beta(6)
\end{gathered}
$$

where $(\alpha, \beta)$ are spherical angles relative to the local magnetic field, $(\theta, \phi)$ are spherical angles relative to the telescope axis, and $A$ is the telescope response function, which has units of area. The integral over $t$ includes only times when the spacecraft $L$, which is a function of $t$, is within the $n$th $L$ shell bin. The simplest way to solve for $j_{1}$ is to assume that within each $i, k, n, m$ interval it is a constant, $j_{i k n m}$. Then

$$
j_{i k n m}=N_{i k n m}\left[2 \pi \int_{\alpha_{1 k}}^{\alpha_{1 k+1}} \int_{E_{i}}^{E_{i+1}}\right.
$$

$\left.\int_{\substack{t_{m} \\ L_{n}<L<L_{n+1}}}^{t_{m+1}} H\left(E, \alpha\left(\alpha_{1}, t\right), \theta_{B}(t)\right) \frac{d \alpha}{d \alpha_{1}} d t d E d \alpha_{1}\right]^{-1}$

where

$$
\begin{aligned}
& H\left(E, \alpha, \theta_{B}\right)=\frac{1}{2 \pi} \\
& \quad \cdot \int_{0}^{2 \pi} A(E, \theta(\alpha, \beta), \phi(\alpha, \beta)) \cos \theta(\alpha, \beta) \sin \alpha d \beta
\end{aligned}
$$

is the effective area of the telescope averaged over the gyrophase angle $\beta . H$ is a function of $E, \alpha$, and the angle $\theta_{B}$ between the telescope axis and the magnetic field. It is related to the standard telescope geometry factor $G$ by

$$
G(E)=2 \pi \int_{0}^{\pi} H\left(E, \alpha, \theta_{B}\right) d \alpha
$$

which is independent of $\theta_{B}$. However, $G$ can only be used when $j$ is isotropic (independent of $\alpha$ ). Note that $H$ is a function of time through $\theta_{B}$, so that the integral of $H$ in (7) takes into account the amount of time spent at each $\theta_{B}$ during the time interval $m$. For example, it is possible that most of the time is spent at a $\theta_{B}$ where $H$ is small, so a small number of counts could still mean a high intensity. Since the spacecraft does not orbit at constant $B / B_{0}$, there is also a time dependence in $\alpha$ when it is translated from $\alpha_{1}$ for evaluation of $H$. This is also taken into account in (7).

To calculate $H$ from (8) we have used a Monte Carlo integration. Particle trajectories were chosen with fixed $\theta_{B}$ and $\alpha$ values, while $\beta$ and the position that they enter the telescope were varied at random. The trajectories were followed through the geometry of MAST until they either stopped, according to a theoretical range-energy relationship, or exited the telescope. Trajectories were included in the integration if they satisfied the same set of selection criteria as was applied to the analysis of the flight data. The values of $\theta_{B}$ and $\alpha$ were then varied in steps of $10^{\circ}$ over the range of possible values. Intermediate values for the integrations in (7) were found by linear interpolation. To test the procedure, we have compared numerical integrations of $H$ according to (9) with a direct Monte Carlo calculation of $G$. The results were consistent within the statistical errors of the Monte Carlo integrations for all $\theta_{B}$ values chosen. Some sample plots of $H$ versus $E$ for oxygen at selected $\alpha$ and $\theta_{B}$ are shown in Figure 6. They illustrate the acceptance cone angle of MAST $\left(\sim 50^{\circ}\right)$ and its convolution with the cone of trajectories at a constant pitch angle $\alpha$. 

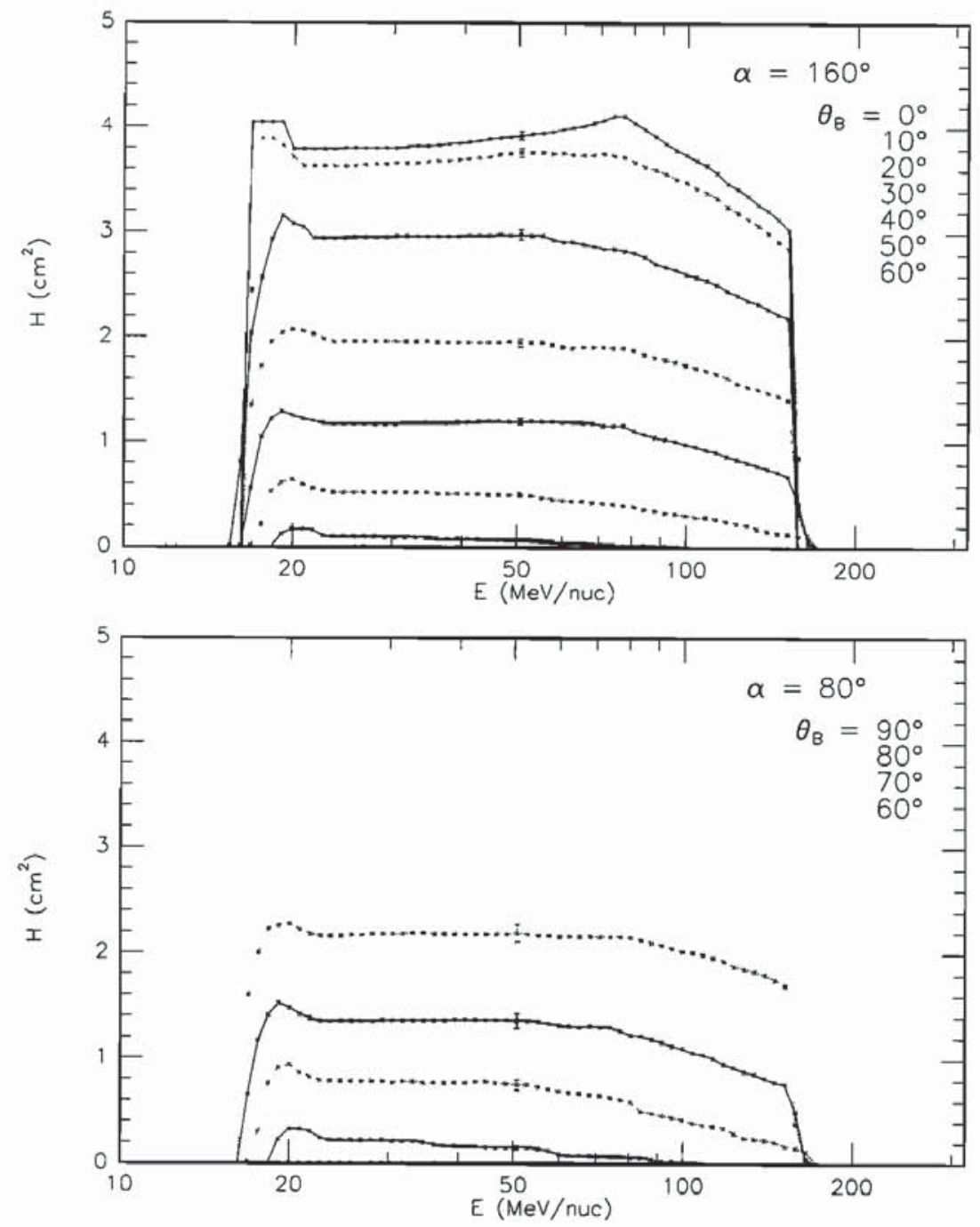

Figure 6. The effective area of MAST averaged over gyrophase, $H$, as a function of energy for oxygen, at various selected values of the telescope angle relative to $\mathbf{B}$ and the particle pitch angle. Normalization errors due to the Monte Carlo integration are shown near the center of each curve.

It is impractical to use (7) directly to calculate $j_{1}$ because it is a function of four variables and the data are limited, so the number of particles in each bin would be small. Instead, we have chosen to represent $j_{1}$ as a separable function

$$
j_{1}\left(E, \alpha_{1}, L, t\right)=U(E, L) V\left(\alpha_{1}\right) W(t)
$$

As is shown in the results below, we found it necessary to maintain the $E$ and $L$ dependences in a single function, but separating the functions of $\alpha_{1}$ and $t$ was a reasonable approximation given the limitations of the data. The three functions $U, V$, and $W$ were calculated as follows: Each function was assumed to be constant within each bin, so that, for example, $V_{k}$ represents the value of $V$ within the $k$ th $\alpha_{1}$ bin. Initial values of $V$ and $W$ were chosen to be 1 for all $\alpha_{1}$ and $t$ bins. An iterative solution was then found by successively evaluating

$$
U_{i n}=\frac{\sum_{k, m} N_{i k n m}}{\sum_{k, m} V_{k} W_{m} \bar{H}_{i k n m}}
$$

$$
\begin{gathered}
V_{k}=\frac{\sum_{i, n, m} N_{i k n m}}{\sum_{i, n, m} U_{i n} W_{m} \bar{H}_{i k n m}} \\
W_{m}=\frac{\sum_{i, k, n} N_{i k n m}}{\sum_{i, k, n} U_{i n} V_{k} \tilde{H}_{i k n m}}
\end{gathered}
$$

where $\bar{H}_{i k n m}$ is the factor in brackets in (7). The sequence (11) was repeatedly evaluated until the values of $U, V$, and $W$ converged. In practice, only a few iterations were required.

\subsection{Results}

The trapped ACRs are observed over a region southeast of the South Atlantic anomaly between the southern tips of South America and Africa [Cummings et al., 1993, 1994]. The intensities of oxygen nuclei observed in this region during a $\sim 20$-month period beginning in July 1992, when SAMPEX was launched, have been 


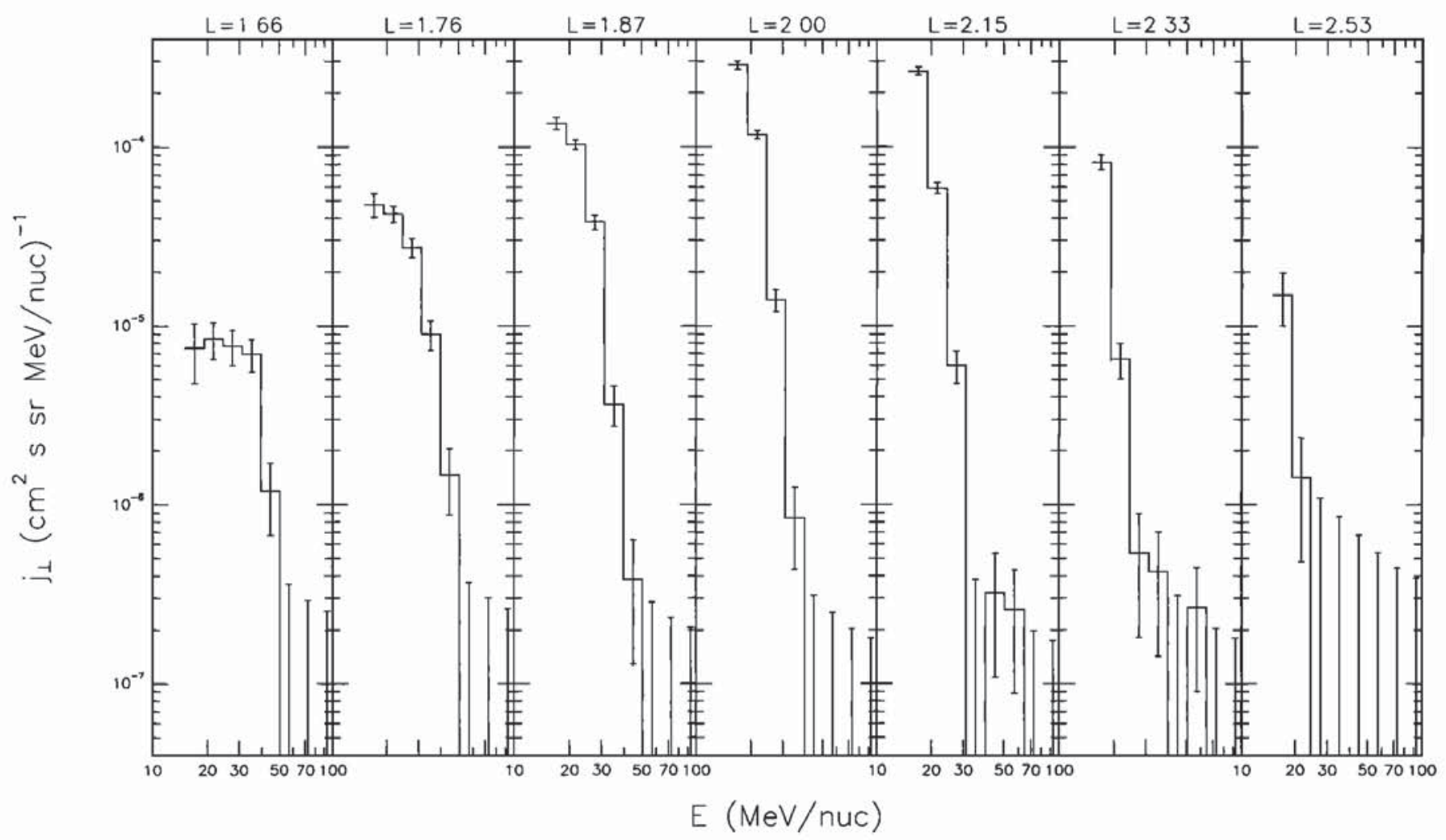

Figure 7. Average energy spectra of trapped $O$ perpendicular to the magnetic field. The data were divided into bins of $2^{\circ}$ invariant latitude, and the $L$ shell at the center of each bin is shown above each spectrum.

evaluated with the method described above. The intensity units were retained in the function $U(E, L)$ which is shown as a function of $E$ and $L$ in Figures 7 and 8 respectively. The functions $V\left(\alpha_{1}\right)$ and $W(t)$ and are shown in Figures 9 and 10 respectively. Due to the normalizations chosen for $V$ and $W, U$ represents the average intensity perpendicular to the magnetic field. Error bars in Figures 7 to 10 are statistical. Systematic errors should be no larger than $\sim 10 \%$, as shown in Figure 6.

Starting with the energy spectra (the $E$ dependence of $U$ ), these were evaluated in eight energy bins logarithmically spaced from 15 to $105 \mathrm{MeV} /$ nucleon. Each spectrum was evaluated over a range in $L$ corresponding to a $2^{\circ}$ change in invariant latitude $\left(\cos ^{-1}\left[L^{-1 / 2}\right]\right)$ from $38^{\circ}$ to $50^{\circ}$. The $L$ value at the center of each $L$ bin is shown above each of the six spectra in Figure 7. The spectra show a general trend of flattening at the lower energies as $L$ decreases. This trend is expected because the cutoff rigidity increases with decreasing $L$, prohibiting access for the lower energy particles. The lower energies then fill in due to energy loss of the trapped particles producing a nearly flat spectrum [Blake, 1990; Tylka, 1994]. The integral energy spectra shown in Figure 5 are consistent with the $L=2$ and $L=2.15$ spectra in Figure 7, where the intensities are greatest.

The data from Figure 7 are also shown as a function of $L$ for four energy intervals in Figure 8. The 15 to $19 \mathrm{MeV} /$ nucleon intensity is seen to be peaked at $L \approx$ 2.1 , while the peak intensity is at lower $L$ values for higher energies, as is expected on the basis of cutoff considerations. The total number of events observed in each $L$ bin is also shown in Figure 8 .

The pitch angle distribution $V\left(\alpha_{1}\right)$ is shown in the top panel of Figure 9. It was calculated for $5^{\circ}$ bins in $\alpha_{1}$ and normalized to the average of the values on each side of $90^{\circ}$. Since the observations were in the southern hemisphere, low and high values of $\alpha_{1}$ correspond to upward and downward trajectories, respectively. The distribution is nearly isotropic in the region outside the atmospheric loss cones, whose edges are at $\sim 65^{\circ}$ to $70^{\circ}$ and $\sim 110^{\circ}$ to $115^{\circ}$. This would be expected if the interplanetary source were constant over a long time period prior to the observations and if the stripping process occurred in a single atomic interaction. Then both the source and loss rates of the trapped ACRs would be proportional to the atmospheric density at the mirror point altitude, so their ratio, which determines the intensity, would be independent of the mirror point and therefore of the pitch angle at a given altitude. If the electron stripping occurred in more than one step, then the probability of stripping and the source strength would be stronger functions of the atmospheric density than the loss rate. The pitch angle distribution would then be peaked near the edges of the loss cone where the mirror points are lowest. The nearly isotropic distribution observed outside the loss cones therefore suggests that the stripping is a single-step process, and this should continue to be true at higher altitudes. However, because the particle lifetimes increase with mirror point altitude, there should perhaps be a local 

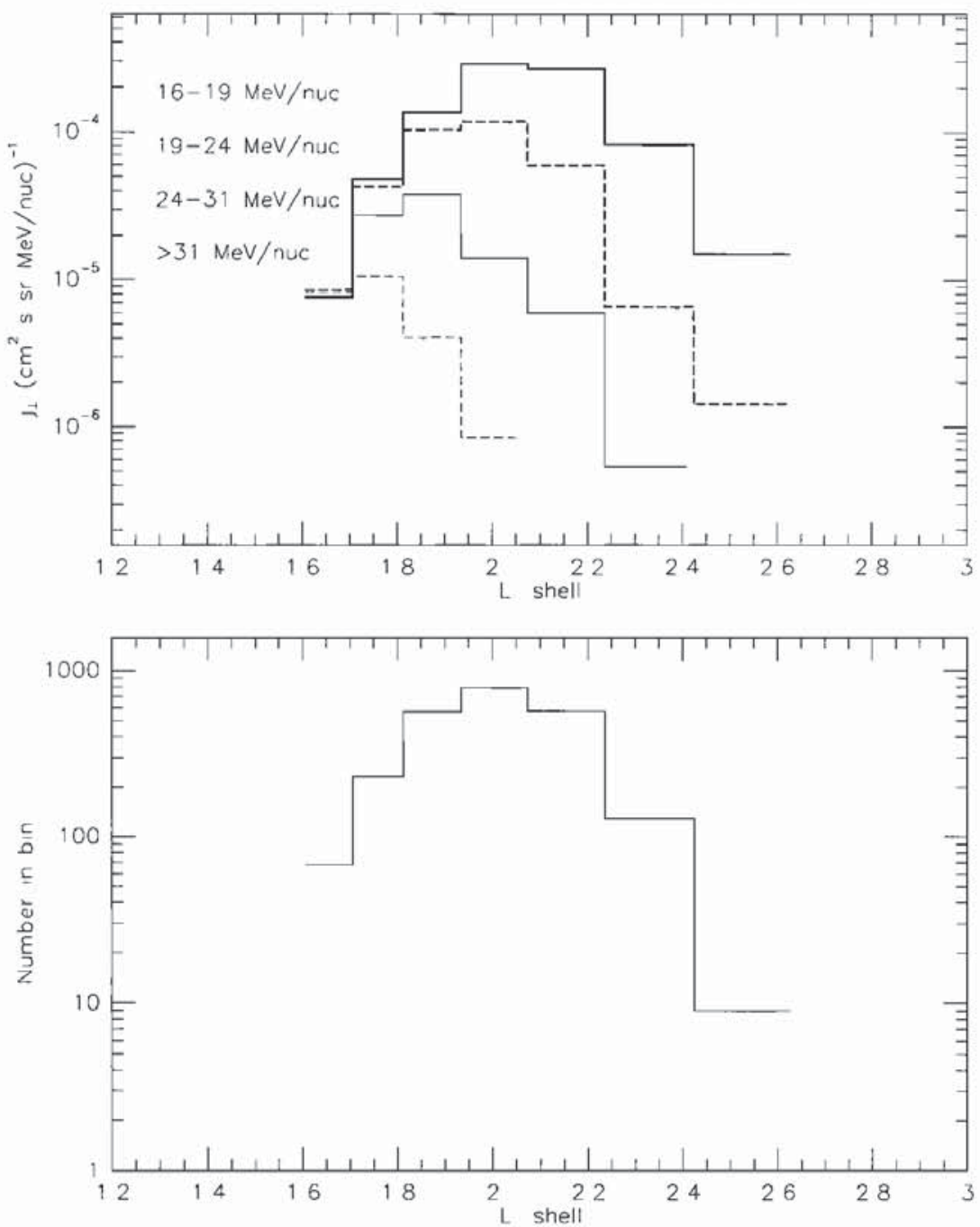

Figure 8. (top) The average intensity versus $L$ shell of $90^{\circ}$ pitch angle trapped $O$ at $R=1.3$ in 4 energy ranges. (bottom) The total number of $O$ nuclei observed in each $2^{\circ}$ invariant latitude $L$ shell bin.

minimum in the pitch angle distribution at $90^{\circ}$ when the interplanetary intensity is increasing with time. There is in fact some indication of such a local minimum in Figure 9.

The pitch angle distribution in Figure 9 shows that the intensities inside the loss cones are consistent with much lower values than those outside, although the upper limits at small pitch angles are relatively high due to the small amount of time that SAMPEX spends pointing down. The intensities in the loss cones, shown by the dotted lines in Figure 9, were not included in the summations of (11a) and (11c), so that they do not contribute to the total intensity, for the following reasons: They are not part of the trapped distribution, and their values are expected to be inaccurate both because of the low number of counts and because a nontrapped distribution will not necessarily be gyrotropic (independent of $\beta$ ) as assumed in the calculation of $H$. The number of counts in each bin are also shown in Figure 9. The double peak is due to the separation of the pitch angles away from $90^{\circ}$ when they are converted from $\alpha$ to $\alpha_{1}$.
The variation of the trapped intensity with time, $W(t)$, is shown in Figure 10 with a similar format to Figure 9. The bins are 15 days wide, and the normalization is to an average value of 1 over the entire period. The number of counts in each bin shows a 3-month periodicity due to the precession of the SAMPEX orbit, which provides more favorable viewing geometry when the orbit is in the noon-midnight plane rather than dawn-dusk. That the periodicity is not evident in the intensity versus time demonstrates that the changes in viewing geometry have been properly compensated for, thereby validating the calculation of the pitch angle distribution. The general trend of increasing intensity is a result of the corresponding increase in the interplanetary ACR intensity with the approach to solar minimum (discussed in more detail in section 5). The decrease in the peak counting rates with time since mid-1993 (bottom panel of Figure 10) is due to an instrumental problem that is corrected for in the data analysis.

The results described above determine the trapped ACR intensity at and below the SAMPEX orbit. It is also possible to place limits on the intensity of trapped 

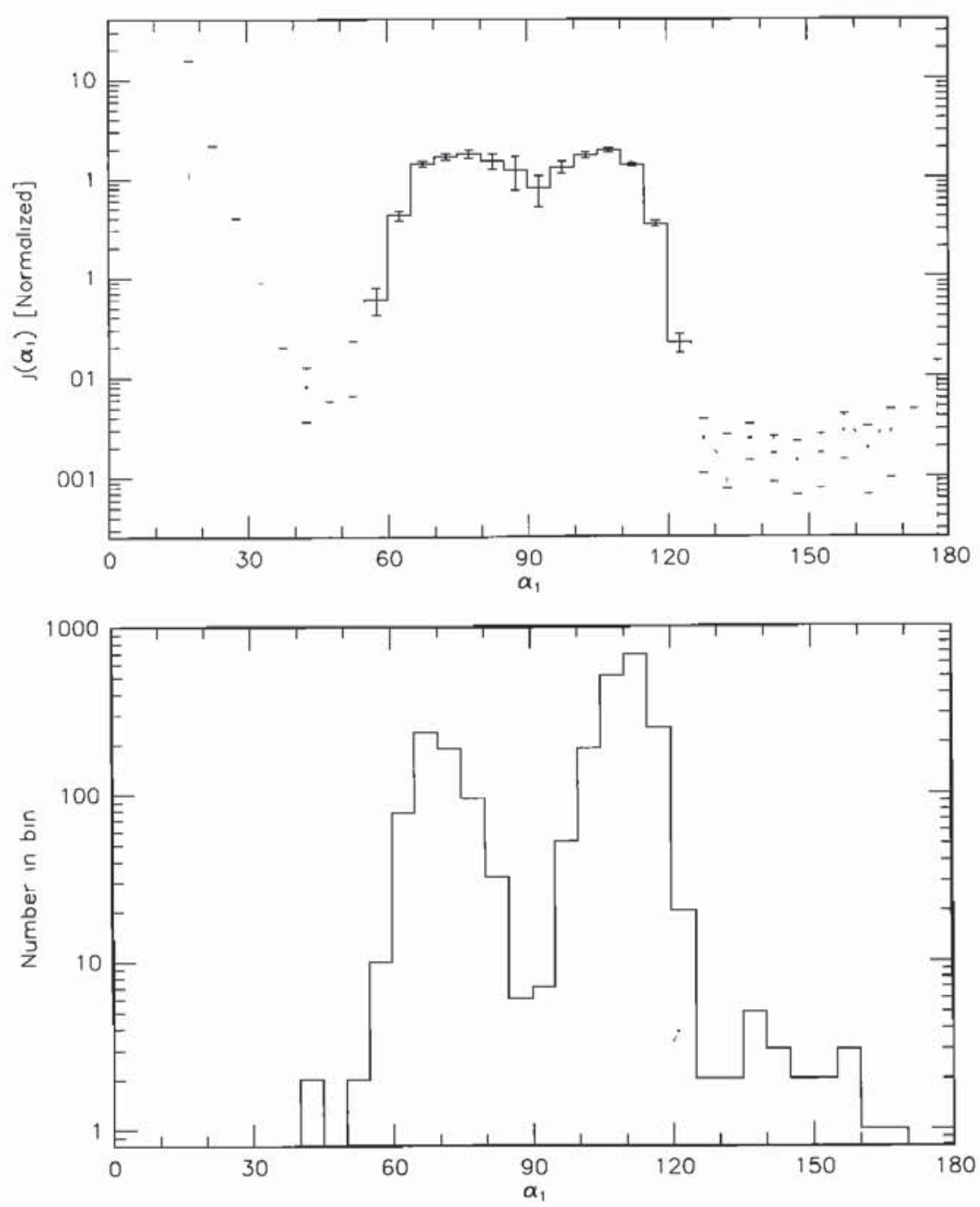

Figure 9. (top) Normalized pitch angle distribution of trapped $O$. The pitch angles were transformed to $R=1.3$ as described in the text. (bottom) The total number of $O$ nuclei observed in each $5^{\circ}$ pitch angle bin.

ACRs that must exist above the SAMPEX orbit. First, we consider the omnidirectional intensity due to only those particles observed by SAMPEX, assuming that there are none with higher mirror points. For $R>1.3$ this represents a lower limit on the omnidirectional intensity. A color coded map of $E>15 \mathrm{MeV} /$ nucleon $\mathrm{O}$ ions in polar $R-\Lambda$ coordinates is shown in Plate 1 . It was obtained by converting the observed pitch angle distribution to each $R$ and $\Lambda$, interpolating the energy spectra between the centers of the $L$ shell bins in Figure 7, averaging over the time variations in Figure 10, and integrating over pitch angle and energy. The approximate $R$ values of the upper atmosphere and the SAMPEX orbit in the south Atlantic region are also shown in the figure. They are significantly greater than 1 because of the low, nondipolar magnetic field in the vicinity of the South Atlantic anomaly. Only particles that mirror between these $R$ values are observed by SAMPEX.

The discussion of the isotropic part of the pitch angle distribution in Figure 9 suggests that the intensity of $90^{\circ}$ pitch angle particles at points above the SAMPEX orbit should be equal to the value observed at the orbit, if their lifetimes are determined solely by energy loss in the atmosphere. Assuming this to be true, an intensity map similar to Plate 1, but calculated with the same perpendicular intensity at all points above $R=1.3$ as observed at $R=1.3$, is shown in Plate 2 . Here the intensity generally increases with $R$ on a given $L$ shell due to the narrowing of the loss cone. Although Plate 2 probably represents a closer approximation than Plate 1 , the true intensity for $R>1.3$ may differ from that shown in Plate 2 due to the long lifetimes of particles that mirror at high altitudes. Their intensity can be reduced by radial diffusion or other loss mechanisms, and they represent a longer time average than at low altitudes so that solar cycle variations will be less apparent.

\section{Relationship to the Interplanetary Source}

We can use a simple model to illustrate the influence of interplanetary conditions on the trapped particle 

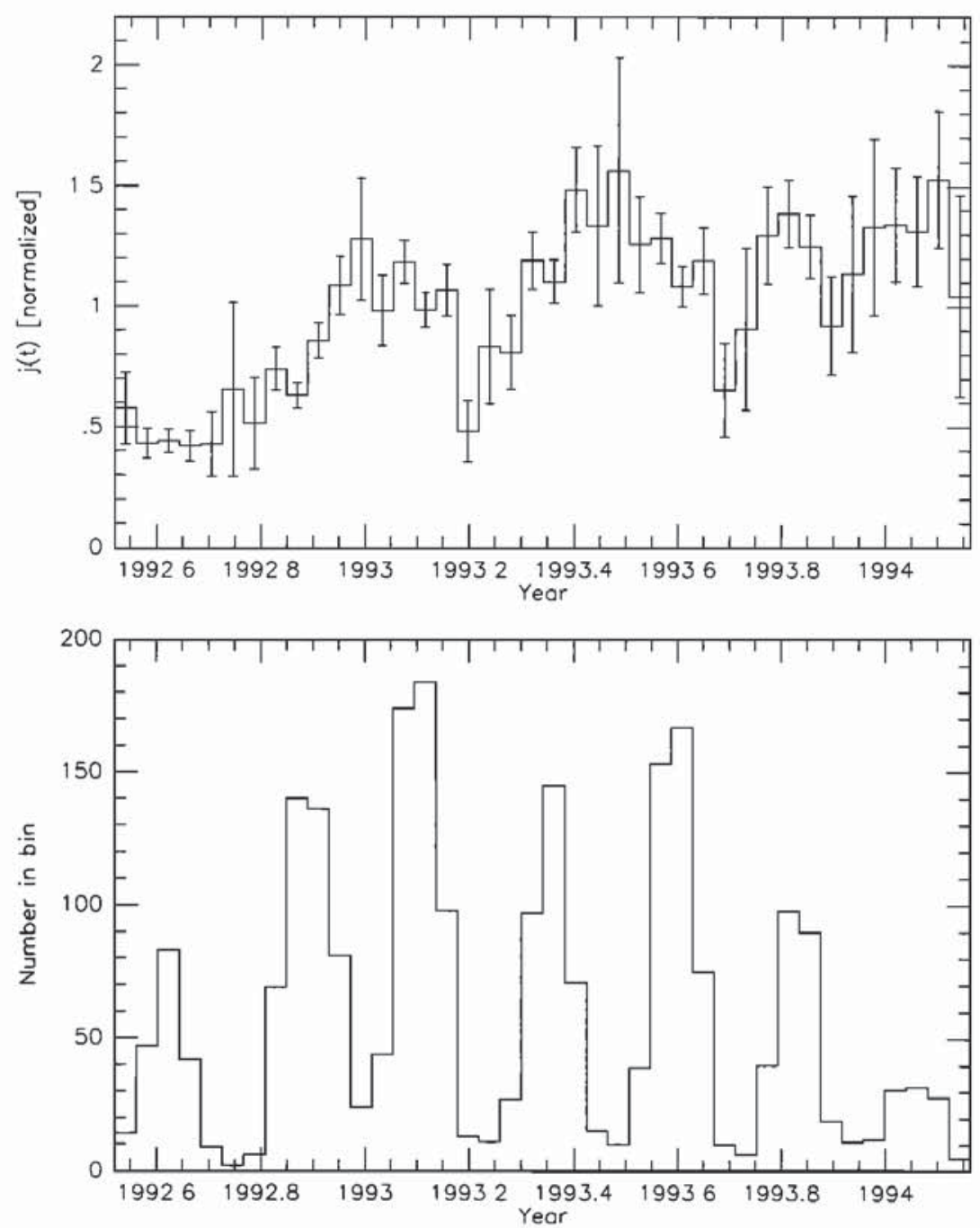

Figure 10. (top) Normalized intensity of trapped $O$ versus time. (bottom) The total number of $\mathrm{O}$ nuclei observed ịn each 15 day time bin.

intensity. The interplanetary ACR intensity $j_{I}(E, t)$ is a function of energy $E$ and time $t$. The initial energy $E_{0}$ at which a particle becomes trapped decreases due to ionization loss in the residual atmosphere near the mirror point until it is removed from the radiation belt after a lifetime $\tau\left(E_{0}\right)$. The energy $E$ at which a trapped particle is observed is therefore related to $E_{0}$ and the earlier time $t_{0}$ at which the particle became trapped. Considering particles that are trapped at a given $L$ and mirror point altitude, their intensity is

$$
j(E, t)=\int_{t-\Delta t_{\max }}^{t-\Delta t_{\text {cow }}} \frac{P\left(E_{0}\right)}{\tau_{B}(E)} j_{I}\left(E_{0}, t_{0}\right) \frac{d E_{0}}{d E} d t_{0}
$$

where $P$ is the probability that the singly ionized interplanetary ACR is stripped of enough electrons to become trapped, and $\tau_{B}(E) \approx 4 L a\left[m_{p} /(2 E)\right]^{1 / 2}$ is the bounce period of the subsequently trapped particle. The time $\Delta t_{\max }$ required for the energy to change from $E_{\max }$ to $E$, where $E_{\max }$ is the maximum trapped particle energy, is $\tau\left(E_{\max }\right)-\tau(E)$. The time $\Delta t_{c w}$ is either zero if $E>E_{c w}$, where $E_{c w}$ is the west cutoff energy, or if $E<E_{c w}$, it is the time required for the energy to change from $E_{c w}$ to $E, \tau\left(E_{c w}\right)-\tau(E)$.

Unfortunately, the variables on the right-hand side of (12) are not well known, and we must make several simplifying assumptions to predict $j(E, t)$. For the interplanetary source intensity we adopt an exponential form, $j_{I}(E, t)=T(t) \exp (-E / \bar{E})$, with $\bar{E}=$ $8 \mathrm{MeV} /$ nucleon (section 3 ), that is approximately valid for the energy range of interest and includes time variability in the normalization factor $T$. To model the energy dependence of the stripping cross section, we set $P(E)=P_{0} \exp \left(-E / E_{P}\right)$, where $E_{P}$ will be adjusted to give the observed trapped particle energy spectrum. In addition, we adopt the approximation that the total distance traveled by a trapped particle with initial energy $E_{0}$ during its lifetime $\tau\left(E_{0}\right)$ is proportional to $E_{0}^{3 / 2}$, which leads to $\left(E_{0}-E\right) / E_{0}=\left(t-t_{0}\right) / \tau\left(E_{0}\right)$, $d E_{0} / d E=1$, and $\tau\left(E_{0}\right)$ proportional to $E_{0}$. If we assume that $T(t)$ varies slowly enough that it can be taken outside the integral in (12), then, expressing $E_{0}$ in terms of $E$ and $t_{0}$ and integrating gives 


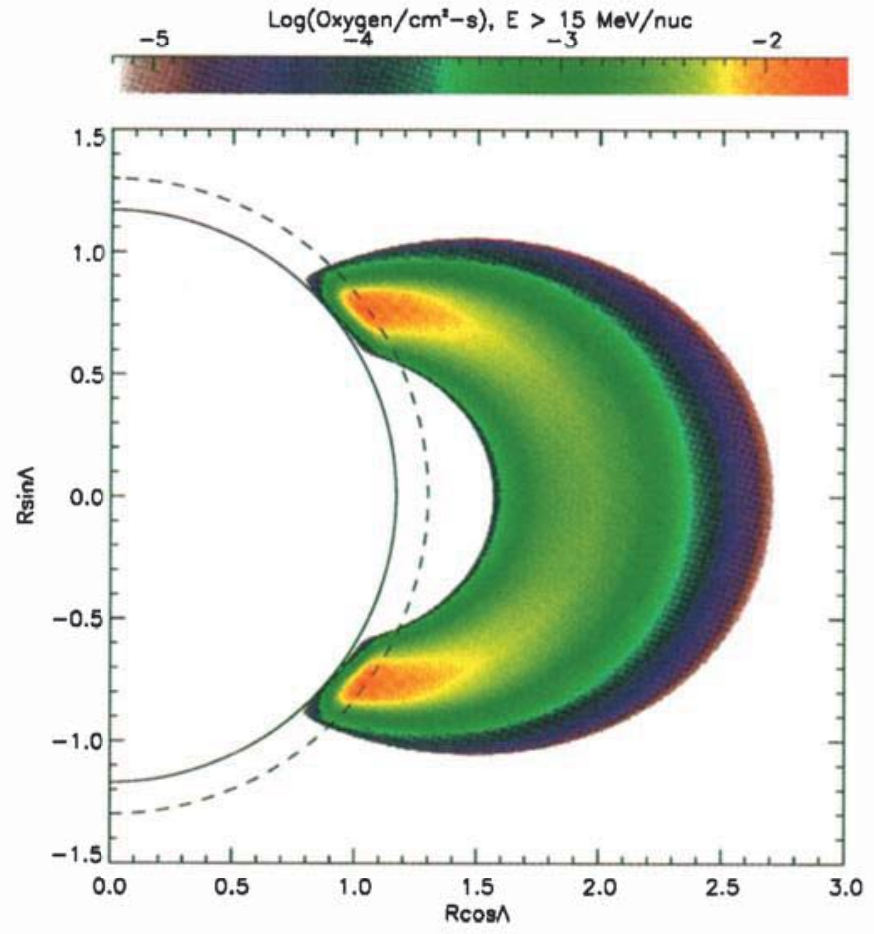

Plate 1. Color-coded omnidirectional intensity map of trapped $O$ in the $R-\Lambda$ polar coordinate system. The solid circle represents the approximate $R$ value of the Earth's upper atmosphere in the South Atlantic region above which the data were taken. The dashed circle represents the approximate $R$ value (1.3) of SAMPEX in this region. Intensities outside the dashed circle represent a lower limit on the true intensity. They were calculated assuming that there are no trapped particles with mirror points in this region.

$$
\begin{aligned}
& j(E, t)=P_{0} e^{-E / E_{1}} T(t) \frac{\tau\left(E_{1}\right)}{\tau_{B}(E)} \\
& \cdot\left[e^{-\Delta t_{c w} / \tau\left(E_{1}\right)}-e^{-\Delta t_{\text {max }} / \tau\left(E_{1}\right)}\right]
\end{aligned}
$$

where $1 / E_{1}=1 / E_{P}+1 / \bar{E}$. This is the form that was used for the simulated $\epsilon Q$ distributions in Figure 2 with the appropriate $E_{1}$ values for each element from the data (Figure 5). Note that the last term in brackets modifies the energy spectrum at high and low energies because $\tau(E)$, which enters through $\Delta t_{c w}$ and $\Delta t_{\max }$, is proportional to $E$.

For $E>E_{c w}$ and $E \ll E_{\max }$ we can neglect the last bracketed term in (13), which leads to

$$
j(E, t) \approx \frac{P(E) \tau\left(E_{1}\right)}{\tau_{B}(E)} j_{I}(E, t)
$$

This shows how the trapped and interplanetary intensities are related at medium energies. Neglecting the weak $E$ dependence of $\tau_{B}, E_{1} \approx 4 \mathrm{MeV} /$ nucleon (Figure 5) and $E_{P} \approx 8 \mathrm{MeV} /$ nucleon.

The interplanetary ACR $O$ intensity measured by SAMPEX is shown in Figure 11. It is generally increasing in a similar way to the trapped ACR O (Figure 10). Comparing the trapped and interplanetary data (Fig- ures 8 and 11), and using the measured energy spectra (Figure 5), the ratio of average intensities, $j / j_{I}$, at $E=16 \mathrm{MeV} /$ nucleon and $L=2$, is $\sim 200$. The bounce period is $\tau_{B} \approx 1 \mathrm{~s}$, so $P(16 \mathrm{MeV} /$ nucleon $) \tau\left(E_{1}\right) \approx$ $200 \mathrm{~s}$. This result applies to the particles that mirror at the SAMPEX altitude of $\sim 600 \mathrm{~km}$. If the lifetime for $E_{1}=4 \mathrm{MeV} /$ nucleon particles is $\sim 10$ days, which is within the large range of possible values estimated by Blake and Friesen [1977], then the trapping probability is $\sim 2 \times 10^{-4}$. The model assumes azimuthal symmetry, so the results can be thought of as drift-averaged values.

A good correlation between time variations in the trapped intensity measured on the Kosmos flights at altitudes from 250 to $400 \mathrm{~km}$ and the interplanetary intensity measured by IMP 8 was also found by Grigorov et al. [1991]. The ratio of the average trapped and interplanetary intensities was estimated by Bobrovskaya et al. [1993] to be $\sim 500$, considerably greater than the SAMPEX value considering the lower Kosmos altitudes. However, the estimated uncertainty in the ratio from the Kosmos data was a factor of $\sim 2$, and those data were taken in the earlier period from 1986 to 1988 and apply to a lower energy interval.

\section{Conclusions}

The measurements of geomagnetically trapped heavy ions by the MAST experiment on SAMPEX strongly support the interpretation that they result from the trapping of anomalous cosmic rays from interplanetary space, and are generally consistent with the trapping mechanism originally proposed by Blake and Friesen [1977], for the following reasons:

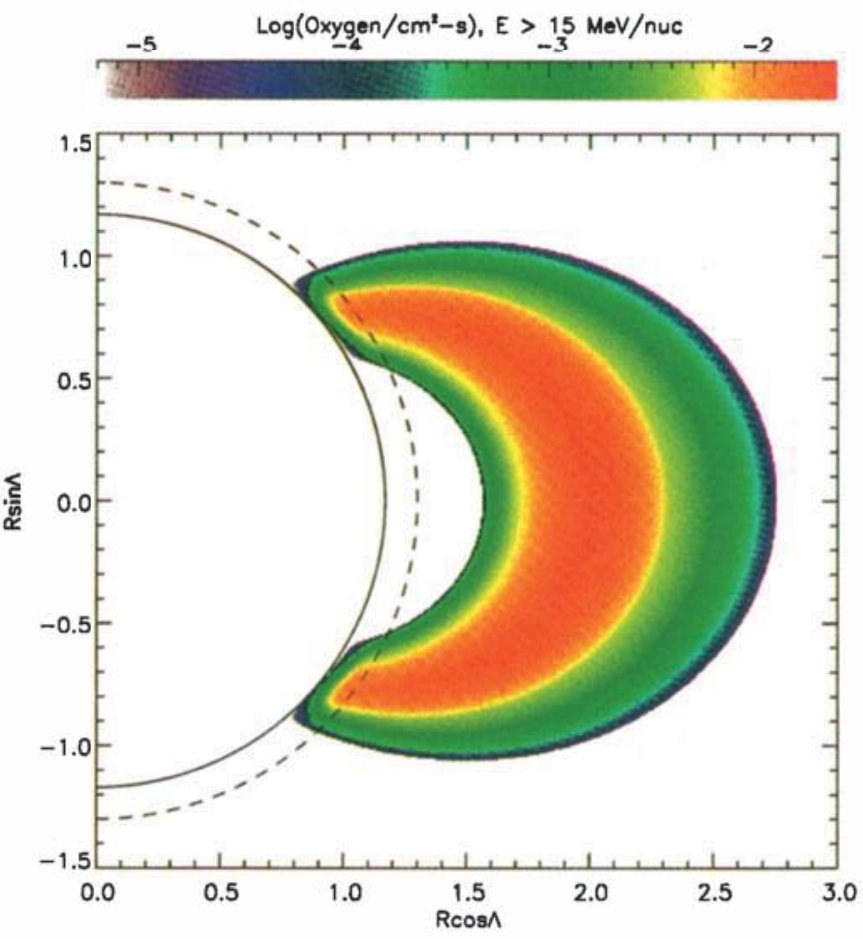

Plate 2. Similar to Plate 1, but the intensities outside the dashed circle $(R=1.3)$ were calculated assuming that the intensities perpendicular to the magnetic field are the same as those observed at the dashed circle. 


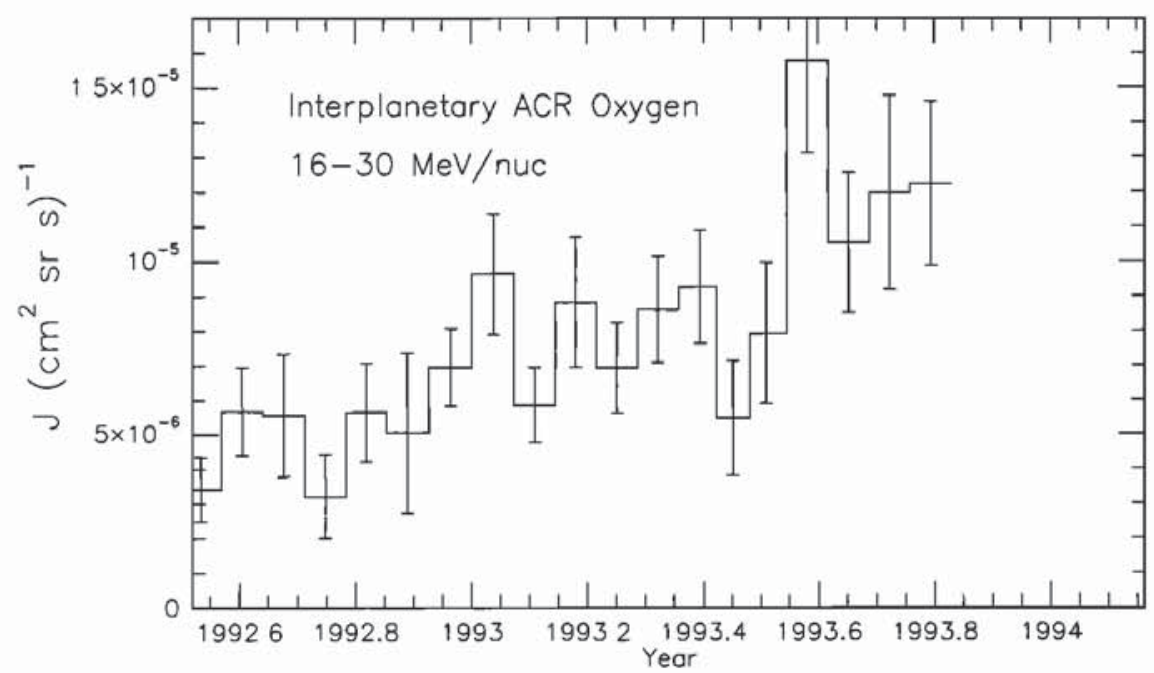

Figure 11. Interplanetary ACR oxygen intensity from 16 to $30 \mathrm{MeV} /$ nucleon measured by SAMPEX. The averaging interval is 26 days.

1. The location of the trapped particle belt is consistent with the expectation that the interplanetary ACRs should penetrate as low in $L$ shell (or latitude) as the western geomagnetic cutoff for singly charged ions and with the requirement that they be adiabatically trapped after removal of electrons by the upper atmosphere.

2. The elements identified in the trapped component $(\mathrm{N}, \mathrm{O}, \mathrm{Ne}$, and possibly $\mathrm{C})$ are also present in the interplanetary ACR component. Of the interplanetary ACR elements that have not been identified as part of the trapped component, $\mathrm{He}$ is expected not to be trapped because of the cutoff and adiabaticity considerations, while $\mathrm{Ar}$ is expected to have its maximum intensity at energies below that covered by MAST [Cummings and Stone, 1990]. There is some evidence for trapped Ar in the Long Duration Exposure Facility (LDEF) data [Jonathal et al., 1993] and in data from the lowenergy ion composition analyzer (LICA) instrument on SAMPEX [Mazur et al., 1993].

3. The relative abundances of the elements are similar to those of the interplanetary ACRs, although the cutoff, adiabaticity, stripping cross sections, and lifetime considerations are expected to modify them somewhat, particularly for C.

4. The energy spectra of the trapped $O$ soften with increasing $L$, as is expected on the basis of the energy dependence of the geomagnetic cutoff and the energy loss of the trapped particles in the residual atmosphere.

5. The pitch angle distribution of the trapped $O$ is nearly isotropic outside the loss cone, with little or no flux in the loss cone. This is consistent with a stably trapped population for which pitch angle scattering is relatively insignificant and with source and loss rates that both vary in proportion to the atmospheric density at the mirror point altitude. Such a source rate is to be expected if the electron-stripping process occurs in a single atomic interaction.

6. The time variations in the trapped intensity are correlated with those of the interplanetary ACRs, the source of the trapped component. At present, the intensities are generally increasing due to the approach of solar minimum.

In addition to the above list of evidence favoring the trapped ACR model, there are several aspects of the measurements that provide further constraints on model parameters or, with proper interpretation, may add new refinements to the model:

1. The outer limit of the trapped component observed by SAMPEX is near an adiabaticity parameter of $\epsilon=$ $0.8 / Q$, where $Q$ is the charge state of the particles when they are initially trapped. Assuming that the outer limit is at a common $\epsilon$ value for all elements, the relative locations of the trapped $\mathrm{N}, \mathrm{O}$, and Ne suggest that initially the particles are not fully stripped and have common average charge states. The low $\mathrm{C}$ abundance should place further constraints on the predominant initial charge state. Since a combination of charge states is likely, further theoretical studies will be required to resolve this issue and to empirically determine the maximum $\epsilon$ value.

2. The differences in the relative abundances and energy spectra of the elements in the trapped and interplanetary populations should also have implications for the adiabatic trapping mechanism. The abundances are still statistically limited, but they do show that the trapped $\mathrm{N}$, possibly $\mathrm{Ne}$, and especially $\mathrm{C}$ are depleted relative to $\mathrm{O}$. The trapped particle energy spectra of $\mathrm{O}$ and $\mathrm{Ne}$ are considerably softer than the corresponding interplanetary spectra, with $e$-folding energies of $\sim 4$ and $8 \mathrm{MeV} /$ nucleon for the trapped and interplanetary spectra, respectively. Also, the trapped spectra soften with increasing atomic number. These properties have not yet been fully explained.

3. The detailed shape of the pitch angle distribution has not yet been predicted theoretically. The observations should provide a useful comparison.

4. A comparison of the trapped and interplanetary intensities can provide a useful test of the theory of ACR 
trapping. At $\sim 600 \mathrm{~km}$ altitude the ratio of the maximum observed trapped intensity to the interplanetary intensity is $\sim 200$ at $16 \mathrm{MeV} /$ nucleon, due to the storage of the trapped particles. Combined with a theory relating the source to the trapped particle intensities, this ratio provides an estimate, $\tau P \sim 200 \mathrm{~s}$, of the product of the average trapped particle lifetime and the probability that an interplanetary ACR mirroring at a given altitude will lose electrons and become trapped. If accurate measurements of the time variability in both the trapped and interplanetary intensities were available, then each of these factors could be determined. Numerical calculations of particle trajectories could provide independent estimates of the lifetimes and, if the stripping cross sections were measured, the trapping probabilities.

Acknowledgments. We thank J. B. Blake for helpful discussions. This work was supported by NASA under contract NAS5-30704 and grant NAGW-1919.

The editor thanks E. Möbius and another referee for their assistance in evaluating this paper.

\section{References}

Adams, J. H., M. Garcia-Munoz, N. L. Grogorov, B. Klecker, M. A. Kondratyeva, G. M. Mason, R. E. McGuire, R. A. Mewaldt, M. I. Panasyuk, Ch. A. Tretyakova, A. J. Tylka, and D. A. Zhuravlev, The charge state of the anomalous component of cosmic rays, Astrophys. J., 375, L45-L48, 1991.

Biswas, S., and N. Durgaprasad, Skylab mearurements of low energy cosmic rays, Space Sci. Rev., 25, 285-327, 1980.

Biswas, S., N. Durgaprasad, J. Nevetia, V. S. Venkatavaradan, J. N. Goswami, U. B. Jayanthi, D. Lalk, and S. K. Mattoo, High fluences of low energy (10-25 MeV/amu) C, N, $O$ and heavier ions at $450 \mathrm{~km}$ altitude; Results from the Skylab experiment, Astrophys. Space Sc., 35, 337-347, 1975.

Blake, J. B., Geomagnetically trapped heavy ions from anomalous cosmic rays, Proc. Int. Cosmic Ray Conf., 21st (7), 30-33, 1990.

Blake, J. B., and L. M. Friesen, A technique to determine the charge state of the anomalous low energy cosmic rays, Proc. Int. Cosmic Ray Conf., 15th (2), 341-346, 1977.

Bobrovskaya, V. V., N. L. Grigorov, M. A. Kondratyeva, M. I. Panasyuk, Ch. A. Tetryakova, D. A. Zuravlev, J. H. Adams, Jr., A. J. Tylka, Cosmos observations of anomalous cosmic ray $\mathrm{N}$ and $\mathrm{Ne}$ in the inner magnetosphere, Proc. Int. Cosmic Ray Conf., 23rd (3), 432-435, 1993.

Chan, J. H., and P. B. Price, Composition and energy spectra of heavy nuclei of unknown origin detected on Skylab, Astrophys. J., 375, L539-L542, 1975.

Christian, E. R., A. C. Cummings, and E. C. Stone, Evidence for anomalous cosmic ray hydrogen, Astrophys. J., 334, L77-L80, 1989.

Cook, W. R., A. C. Cummings, J. R. Cummings, T. L. Garrard, B. Kecman, R. A. Mewaldt, R. S. Selesnick, E. C. Stone, and T. T. von Rosenvinge, MAST: A mass spectrometer telescope for studies of the isotopic composition of solar, anomalous, and galactic cosmic ray nuclei, IEEE Trans. Geosci. Remote Sens., 31, 557-564, 1993.

Cummings, A. C., and E. C. Stone, Elemental composition of the very local interstellar medium as deduced from observations of anomalous cosmic rays, Proc. Int. Cosmic Ray Conf., 21st (6), 202-205, 1990.

Cummings, A. C., E. C. Stone, and W. R. Webber, Evidence that the anomalous cosmic-ray component is singly ionized, Astrophys. J., 287, L99-L103, 1984.

Cummings, J. R., A. C. Cummings, R. A. Mewaldt, R. S. Selesnick, E. C. Stone, and T. T. von Rosenvinge, New evidence for geomagnetically trapped anomalous cosmic rays, Geophys. Res. Lett., 20, 2003-2006, 1993.

Cummings, J. R., A. C. Cummings, R. A. Mewaldt, R. S. Selesnick, E. C. Stone, and T. T. von Rosenvinge, SAMPEX observations of geomagnetically trapped anomalous cosmic rays, in Proceedings of the 23rd International Cosmic Ray Conference, Invited, Rapporteur and Highlight Papers, edited by D. A. Leahy, R. B. Hicks, and D. Venkatesan, pp. 475-482, World Scientific, Singapore, 1994.

Fanselow, J. L., and E. C. Stone, Geomagnetic cutoffs for cosmic-ray protons for seven energy intervals between $\mathbf{1 . 2}$ and $39 \mathrm{MeV}$, J. Geophys. Res., 77, 3999-4009, 1972.

Fisk, L. A., B. Kozlovsky, and R. Ramaty, An interpretation of the observed oxygen and nitrogen enhancements in low energy cosmic rays, Astrophys. J., 190, L35-L38, 1974.

Garcia-Munoz, M., G. M. Mason, and J. H. Simpson, A new test for solar modulation theory: The 1972 May-July low energy galactic cosmic ray proton and helium spectra, Astrophys. J., 182, L81-L84, 1973.

Geiss, J., G. Gloeckler, U. Mall, R. von Steiger, A. B. Galvin, and $\mathrm{K}$. W. Ogilvie, Interstellar oxygen, nitrogen and neon in the heliosphere, Astron. Astrophys., 282, 924-933, 1994.

Grigorov, N. L., M. A. Kondratyeva, M. I. Panasyuk, Ch. A. Tretyakova, J. H. Adams Jr., J. B. Blake, M. Schulz, R. A. Mewaldt, and A. J. Tylka, Evidence for anomalous cosmic ray oxygen ions in the inner magnetosphere, Geophys. Res. Lett., 18, 1959-1962, 1991.

Hovestadt, D. O., O. Volmer, G. Gloeckler, and C. Y. Fan, Differential energy spectra of low-energy $(<8.5 \mathrm{MeV}$ per nucleon) heavy cosmic rays during solar quiet times, Phys. Rev. Lett., 31, 650, 1973.

Ilyan, V. D., I. V. Ilyan, and S. N. Kusnetsov, Stochastic instability of charged particles in a geomagnetic trap, Cosmic Res., Engl. Transl., 24, 88-96, 1986.

Jonathal, D., R. Beaujean, and W. Enge, Trapped iron measured on LDEF, Proc. Int. Cosmic Ray Conf., 23rd (3), 445-448, 1993.

Klecker, B., D. Hovestadt, M. Scholer, J. B. Blake, M. C. McNab, G. M. Mason, and J. E. Mazur, Determination of the ionic charge state of the anomalous component of cosmic rays with SAMPEX (abstract), EOS Trans. $A G U$, Spring Meeting, 294, 1994.

Kleis, T., A. J. Tylka, P. R. Boberg, J. H. Adams, Jr., L. P. Beahm, Characteristics of low energy ions in the heavy ions in space (HIIS) experiment, paper presented at the third LDEF post-retrieval symposium, Williamsburg, Va, November 8-12, 1993.

Luhn, A., B. Klecker, D. Hovestadt, G. Gloeckler, F. M. Ipavich, M. Scholer, C. Y. Fan, and L. A. Fisk, Ionic charge states of $\mathrm{N}, \mathrm{Ne}, \mathrm{Mg}, \mathrm{Si}$, and $\mathrm{S}$ in solar energetic particle events, Adv. Space Res., 4, 161-164, 1984.

Mazur, J. E., G. M. Mason, D. C. Hamilton, and J. B. Blake, Low energy trapped anomalous cosmic rays observed with SAMPEX (abstract), EOS Trans. AGU, Fall Meeting, $517,1993$.

McDonald, F. B., B. J. Teegarden , J. H. Trainor, and W. R. Webber, The anomalous abundance of cosmic ray nitrogen and oxygen nuclei at low energies, Astrophys. J., 185, L105-L108, 1974.

Mewaldt, R. A., A. C. Cummings, J. R. Cummings, E. C. Stone, B. Klecker, D. Hovestadt, M. Scholer, G. M. Mason, 
J. E. Mazur, D. C. Hamilton, T. T. von Rosenvinge, and J. B. Blake, The return of the anomalous component to 1 AU in 1992, Geophys. Res. Lett., 20, 2263-2266, 1993.

Pesses, M. E., J. R. Jokipii, and D. Eichler, Cosmic ray drift, shock wave acceleration, and the anomalous component of cosmic rays, Astrophys. J., 246, L85-L88, 1981.

Roederer, J. G., Dynamics of Geomagnetically Trapped Radiation, Springer, New York, 1970.

Schulz, M., The magnetosphere, in Geomagnetism, vol. 4, edited by J. A. Jacobs, pp. 87-293, Academic Press, San Diego, Calif., 1991.

Schulz, M., and L. J. Lanzerotti, Particle Diffusion in the Radiation Belts, Springer, New York, 1974.

Selesnick, R. S., A. C. Cummings, J. R. Cummings, R. A. Leske, R. A. Mewaldt, E. C. Stone, and T. T. von Rosenvinge, Coronal abundances of neon and magnesium isotopes from solar energetic particles, Astrophys. J., 418, L45-L48, 1993.

Singh, R. K., M. Banashree, N. Durgaprasad, S. Biswas, M. N. Vahia, J. S. Yadav, A. Dutta, and J. N. Goswami, Ionization states of the anomalous cosmic rays, Astro. phys. J., 374, 753-765, 1991.
Smart, D. F., and M. A. Shea, The change in geomagnetic cutofts due to changes in the dipole equivalent of the Earth's magnetic field, Proc. Int. Cosmic Ray Conf., 23rd (3), 781-784, 1993.

Spjeldvik, W. N., Expected charge states of energetic ions in the magnetosphere, Space Sc. Rev. 29, 499-538, 1979.

Tylka, A. J., Theoretical modeling and interpretation of trapped anomalous cosmic rays, in Proceedings of the 23rd International Cosmic Ray Conference, Invited, Rapporteur and Highlight Papers, edited by D. A. Leahy, R. B. Hicks, and D. Venkatesan, pp. 465-474, World Scientific, Singapore, 1994.

A. C. Cummings, J. R. Cummings, R. A. Mewaldt, R. S. Selesnick, and E. C. Stone, California Institute of Technology, mail code 220-47, Pasadena, CA 91125. (e-mail: rss@citsrl.caltech.edu)

T. T. von Rosenvinge, NASA Goddard Space Flight Center, Greenbelt, MD 20771.

(Received May 26, 1994; revised November 28, 1994; accepted November 30, 1994.) 